\title{
2-Deoxy-D-glucose enhances the anti-cancer effects of idarubicin on idarubicin-resistant P388 leukemia cells
}

\author{
TAISUKE MATSUO, YUMIKO KONYA, ERI HIRAYAMA and YASUYUKI SADZUKA \\ Division of Advanced Pharmaceutics, Department of Clinical Pharmaceutical Sciences, \\ School of Pharmacy, Iwate Medical University, Iwate 028-3694, Japan
}

Received October 28, 2019; Accepted February 18, 2020

DOI: $10.3892 / \mathrm{ol} .2020 .11616$

\begin{abstract}
Cancer cells switch from mitochondrial oxidative phosphorylation to glycolysis, even in the presence of normal oxygen concentrations. Inhibition of the glycolytic pathway is therefore a critical strategy in cancer therapy. A non-metabolic glucose analog, 2-deoxy-D-glucose (2-DG), has been the focus of research on glycolytic inhibitors for use in cancer treatment. The current study examined the anti-cancer effects of 2-DG on idarubicin (IDA)-resistant P388 (P388/IDA) leukemia cells. P388/IDA cells were established following continuous exposure of IDA to P388 cells. Characterization of P388/IDA cells revealed increased lactate production and glucose consumption compared with P388 parent cells. The results of a cell viability assay determined that 2-DG induces higher toxicity in P388/IDA cells compared with P388 cells. Although 2-DG also exhibits endoplasmic reticulum (ER) stress-inducing activity, the cytotoxic effect of the ER stress inducer, tunicamycin, on P388/IDA cells was lower than that of P388 cells. A combination of 2-DG and IDA enhanced P388/IDA cell death compared with each agent alone. The results indicated that P388 cells activated glycolysis after acquiring IDA resistance and therefore, inhibition of the glycolytic pathway via 2-DG might be a useful strategy for cancer therapy against IDAresistant leukemia cells.
\end{abstract}

Correspondence to: Dr Taisuke Matsuo, Division of Advanced Pharmaceutics, Department of Clinical Pharmaceutical Sciences, School of Pharmacy, Iwate Medical University, 1-1-1 Idaidori, Yahaba-cho, Shiwa-gun, Iwate 028-3694, Japan

E-mail: tmatsuo@iwate-med.ac.jp

Abbreviations: 2-DG, 2-deoxy-D-glucose; ALL, acute lymphoblastic leucemia; AML, acute myelogenous leucemia; DNR, daunorubicin; DOX, doxorubicin; ER, endoplasmic reticulum; IDA, idarubicin; P-gp, P-glycoprotein; Tm, tunicamycin

Key words: 2-deoxy-D-glucose, leukemia, glycolysis, apoptosis

\section{Introduction}

Anthracycline anticancer drugs, such as doxorubicin (DOX), daunorubicin (DNR), and idarubicin (IDA), are broadly used in the treatment of various cancers, including acute leukemia (1-3). These drugs mediate cancer cell death through intercalation between DNA base pairs and inhibition of topoisomerase II (1-3). One of the limitations associated with DOX and DNR therapy is the development of drug resistance through overexpression of the drug transporter such as P-glycoprotein (P-gp) (4-6). On the other hand, IDA, which is used in acute myelogenous leukemia (AML) and acute lymphoblastic leukemia (ALL) therapy, is highly lipophilic compared to DOX and DNR, and is imported into cells faster than the aforementioned anthracyclines, as well as less affected by P-gp-mediated drug efflux $(7,8)$. However, AML and ALL therapy with IDA also face the limitation of drug resistance.

One of the characteristics of cancer cells is their metabolic alteration, known as the Warburg effect $(9,10)$. The rapidly proliferating cancer cells predominantly use the less efficient aerobic glycolytic pathway for ATP synthesis, resulting in high glucose demand in cancer cells. Therefore, inhibition of glycolysis is expected to have a stronger impact on cancer cells than on normal cells. Much research has focused on glycolysis inhibition as a strategy for cancer therapy $(11,12)$.

The non-metabolic glucose analog 2-deoxy-D-glucose (2-DG) is a glycolysis inhibitor (12). 2-DG is transported into the cells and metabolized by hexokinase to 2-deoxy-D-glucose-6-phosphate, which is not further metabolized and accumulates in the cells (12). The structure of 2-DG is similar to that of mannose, which is important for $\mathrm{N}$-glycosylation in proteins and normal protein folding in the endoplasmic reticulum (ER). It has been reported that inhibition of N-glycosylation by its inhibitor Tunicamycin (Tm) induced ER stress (13). Inhibition of N-glycosylation by 2-DG also increases misfolded proteins in the ER and results in ER stress-induced cell death (14). It has been thought that this dual effect of 2-DG provokes cell death and suppresses cell proliferation in cancer cells. Therefore, it is relevant to understand the potential of 2-DG in the mitigation of IDA-resistance in the context of leukemia therapy. In this study, the cytotoxic effect of 2-DG on in-house established IDA-resistant P388 (P388/IDA) leukemia cells was evaluated. 

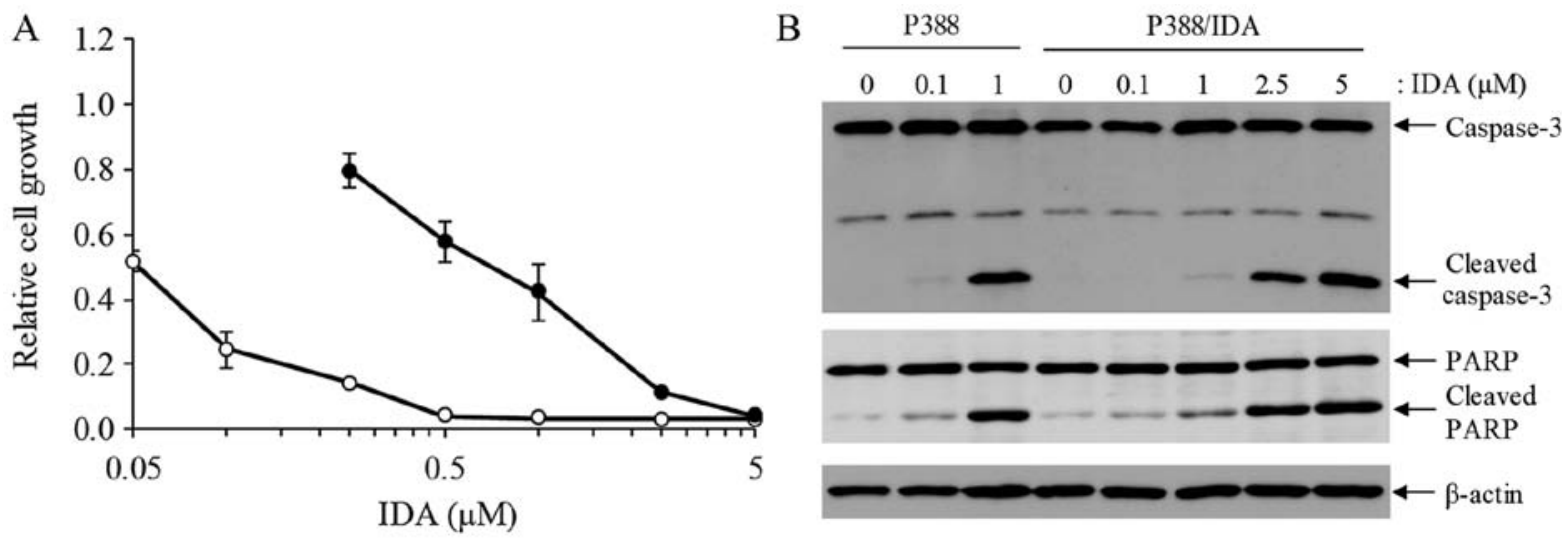

Figure 1. Characterization of P388/IDA cells. P388 and P388/IDA cells were treated with IDA (0-5 $\mu \mathrm{M})$. (A) Cell viability was determined by measuring dehydrogenase activity. P388 and P388/IDA cells are represented by empty and filled circles, respectively. (B) Cleaved PARP and cleaved caspase-3 protein expressions were determined via western blot analysis. Data are presented as the mean \pm standard deviation $(n=4)$. IDA, idarubicin; PARP, poly(ADP-ribose) polymerase.

\section{Materials and methods}

Materials. P388 mouse leukemia cells (P388 cells), which has been used for model of leukemia cells (15-17), were provided by Daiichi Pharmaceutical Co., Ltd. (Tokyo, Japan). RPMI-1640 medium was purchased from Nissui Pharmaceutical Co., Ltd. (Tokyo, Japan). 2-DG (D6134) and tunicamycin (Tm; T7765) were purchased from Sigma-Aldrich (St. Louis, MO, USA). IDA was obtained from Wako (Tokyo, Japan). The Cell Counting Kit-8 (CK04), Lactate Assay Kit-WST (L256), and Glucose Assay Kit-WST (G264) were purchased from Dojindo (Kumamoto, Japan). Anti-caspase-3 (19677-1-AP), anti-Poly (ADP-ribose) polymerase (PARP; 9542), and anti- $\beta$-actin (A5441) antibodies were purchased from Proteintech (Rosemont, IL, USA), Cell Signaling Technology (MA, USA), and Sigma-Aldrich, respectively.

Cell culture and establishment of IDA-resistant P388 cells. P388 leukemia cells were grown in RPMI-1640 medium that contained $50 \mu \mathrm{M}$ 2-mercaptoethanol and $10 \%$ fetal bovine serum, under $5 \% \mathrm{CO}_{2}$ at $37^{\circ} \mathrm{C}$. To establish P388/IDA cells, at first, P388 cells were cultured with $0.001 \mu \mathrm{M}$ IDA for 1 week in 12-well plates. Cells underwent culture with increasingly higher concentrations (1.5 to 2-fold) of IDA every 1-2 weeks. Finally, P388/IDA cells were maintained in RPMI-1640 medium with $0.1 \mu \mathrm{M}$ IDA. In total, the establishment of the drug-resistant cells took more than 6 months. When the cells were used for analyses, the cells were cultured in drug-free medium for more than 3 days.

Measurement of $\mathrm{pH}$, lactic acid production, and glucose consumption. P388 or P388/IDA cells $\left(1.0 \times 10^{6}\right.$ cells $\left./ \mathrm{ml}\right)$ were cultured in RPMI-1640 medium for $24 \mathrm{~h}$ in 6-well plates. The $\mathrm{pH}$ of each medium was measured by LAQUAtwin (Horiba, Ltd., Kyoto, Japan). The pH of RPMI-1640 medium before culturing was 7.9. Lactic acid production and glucose consumption were measured by Lactate Assay Kit-WST and Glucose Assay Kit-WST, respectively, according to the manufacturer's instructions. In brief, cultured media were centrifuged at $10,000 \mathrm{x}$ g for $3 \mathrm{~min}$ and the supernatants were used for analysis. The respective reaction buffers were incubated with the supernatants for $30 \mathrm{~min}$ at $37^{\circ} \mathrm{C}$, and then the absorbance was measured at $450 \mathrm{~nm}$. Lactic acid concentrations were calculated using a lactic acid standard and then normalized to cell number. Glucose concentrations in culture media were calculated using a glucose standard. To determine relative glucose consumption per cell, glucose concentration after culturing was subtracted from that before culturing and then normalized to cell number.

Cell viability. $\mathrm{P} 388$ or $\mathrm{P} 388 / \mathrm{IDA}$ cells $\left(1.5 \times 10^{5}\right.$ cells $\left./ \mathrm{ml}\right)$ were cultured in RPMI-1640 with IDA $(0-5 \mu \mathrm{M}), 2-\mathrm{DG}(0-500 \mu \mathrm{M})$, or Tm $(0-1 \mu \mathrm{g} / \mathrm{ml})$ for $48 \mathrm{~h}$ in 96 -well plates. Cell viability was measured by Cell Counting Kit- 8 according to the manufacturer's instructions.

Western blot analysis. $\mathrm{P} 388$ or P388/IDA cells $\left(1.5 \times 10^{5}\right.$ cells $\left./ \mathrm{ml}\right)$ were treated with IDA $(0-5 \mu \mathrm{M})$ and/or 2-DG $(250 \mu \mathrm{M})$ for $24 \mathrm{~h}$ in 12-well plates. Western blot analysis was performed as previously described (18). In brief, proteins were separated on $7.5 \%$ or $15 \%$ acrylamide gels for SDS-PAGE and transferred to nitrocellulose membranes. Membranes were incubated with anti-caspase-3 (1:1,000), anti-PARP (1:1,500), and anti- $\beta$-actin $(1: 5,000)$ antibodies. Membranes were revealed with horseradish peroxidase-conjugated secondary antibodies (anti-mouse or anti-rabbit $\mathrm{IgG}$ ).

Statistical analysis. Data are presented as the mean \pm standard deviation and were analyzed using a Student's t-test or One-way ANOVA (post hoc test, Tukey Kramer method). BellCurve for Excel version 3.20 was used for statistical analysis (Social Survey Research Information Co., Ltd.). P<0.05 was considered statistically significant.

\section{Results}

Characterization of IDA-resistant P388 leukemia cells. To compare the sensitivity of P388 and P388/IDA cells to IDA, a cell viability assay was performed $48 \mathrm{~h}$ after IDA treatment in both cell lines. The $\mathrm{IC}_{50}$ of IDA on P388 was $0.05 \pm 0.02 \mu \mathrm{M}$, whereas on P388/IDA cells was $0.66 \pm 0.18 \mu \mathrm{M}(\mathrm{P}<0.05)$. IDA sensitivity of P388/IDA cells was approximately 10-fold lower than that of P388 cells (Fig. 1A). Western blot analysis showed that the apoptosis markers cleaved caspase- 3 and cleaved 

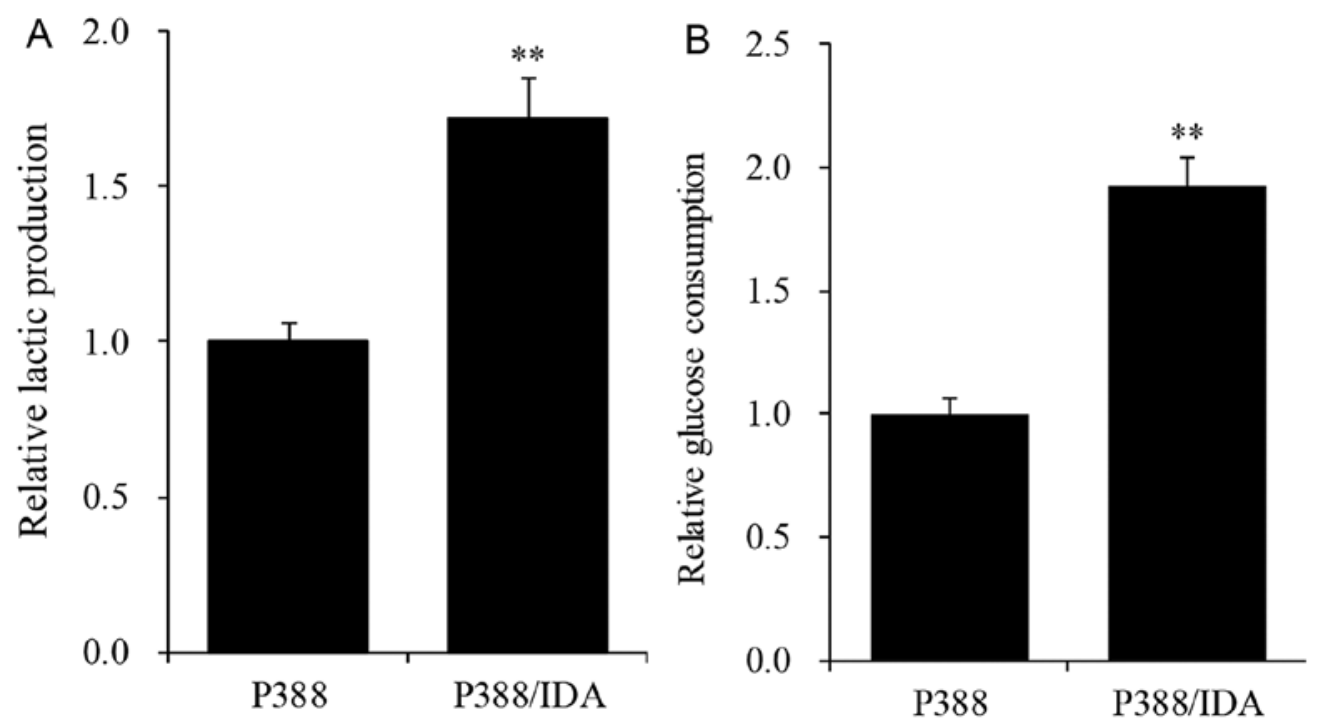

Figure 2. Metabolic alterations in P388/IDA cells. (A) The concentration of lactic acid in culture medium (presented as relative lactic acid production) and (B) glucose consumption (presented as relative glucose consumption) in cells were measured $24 \mathrm{~h}$ following culture. Data are presented as the mean \pm standard deviation ( $\mathrm{n}=3) .{ }^{* *} \mathrm{P}<0.01$ vs. $\mathrm{P} 388$. IDA, idarubicin.
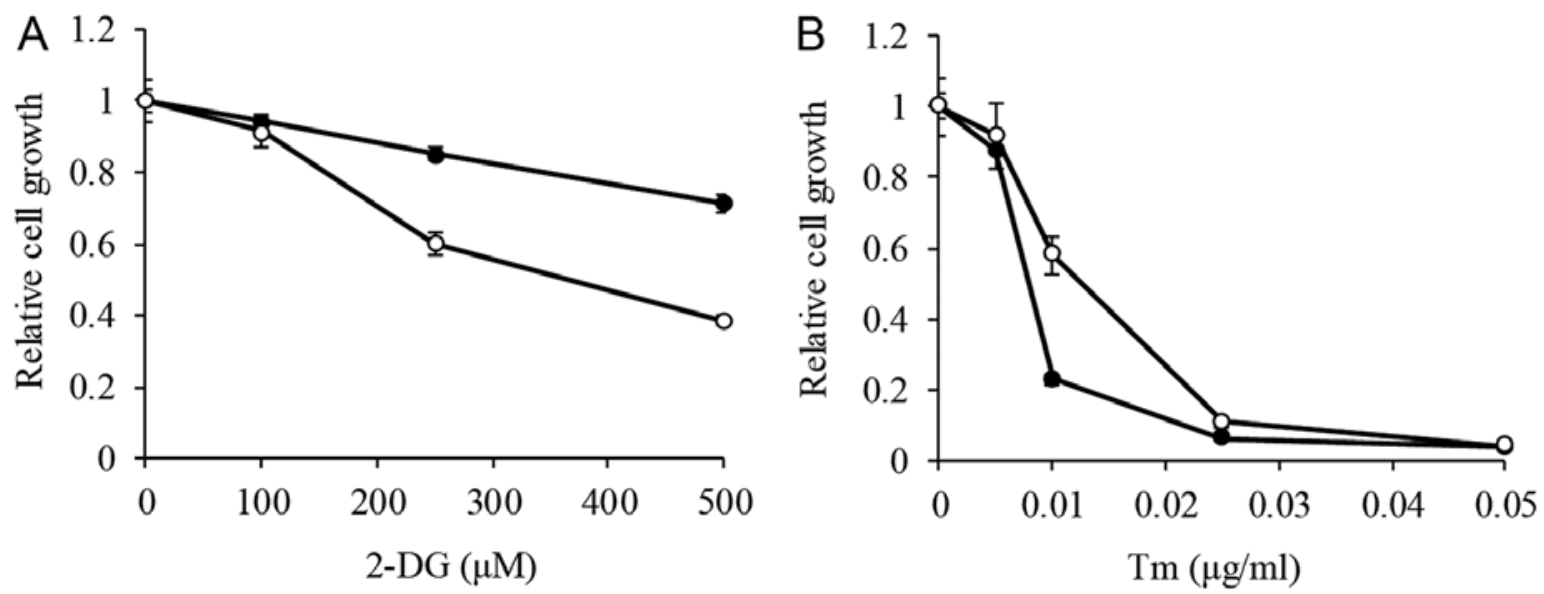

Figure 3. 2-DG and Tm cytotoxicity in P388 and P388/IDA cells. Cell viability in P388 (black circles) and P388/IDA (white circles) cells was determined following treatment with (A) 2-DG and (B) Tm for $48 \mathrm{~h}$. Data are presented as the mean \pm standard deviation (n=4). 2-DG, 2-deoxy-D-glucose; Tm, Tunicamycin; IDA, idarubicin.

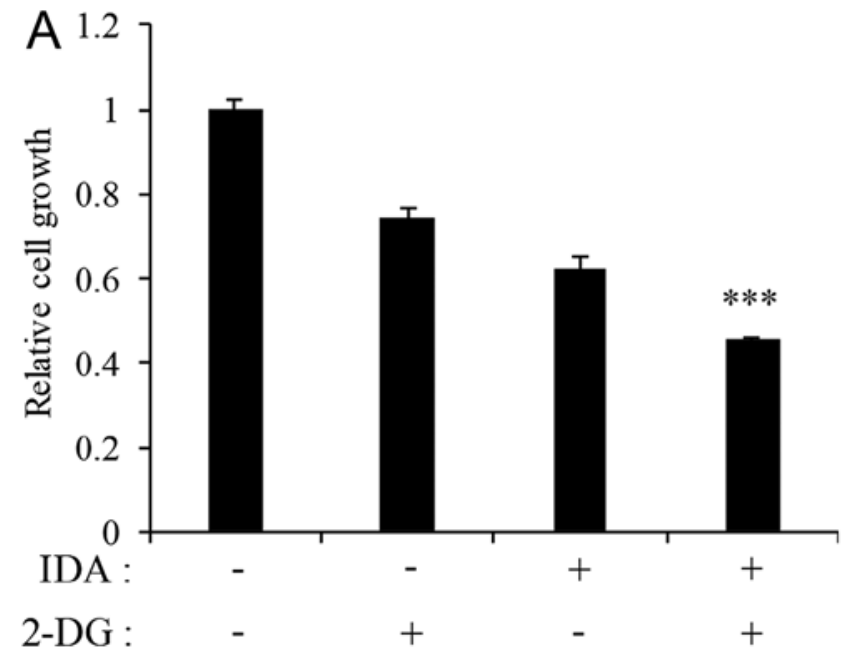

$\mathrm{B}$

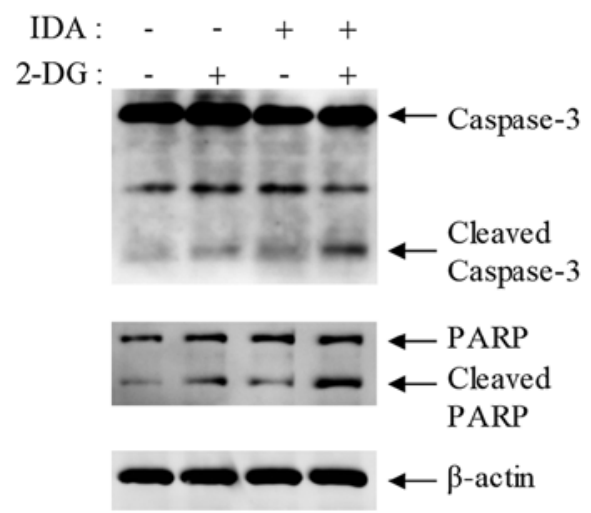

Figure 4. 2-DG and IDA combined treatment in P388/IDA cells. P388/IDA cells were treated with 2-DG ( $250 \mu \mathrm{M})$ and/or IDA (0.5 $\mu \mathrm{M})$ for $48 \mathrm{~h}$. (A) Cell viability was analyzed. (B) Western blot analysis of cleaved PARP and cleaved caspase-3 protein expression. Data are presented as the mean \pm standard deviation ( $\mathrm{n}=4$ ). ${ }^{* * *} \mathrm{P}<0.001$ vs. 2-DG or IDA treatment alone. 2-DG, 2-deoxy-D-glucose; IDA, idarubicin; PARP, poly(ADP-ribose) polymerase. 
PARP were detected in P388/IDA cells undergoing treatment with high concentrations of IDA (Fig. 1B).

To examine the metabolism of IDA/P388 cells, we measured the $\mathrm{pH}$ of culture media $24 \mathrm{~h}$ after incubation. The $\mathrm{pH}$ in $\mathrm{P} 388$ and P388/IDA cells was $7.4 \pm 0.1$ and $6.9 \pm 0.1$, respectively. Lactic acid production in IDA/P388 cells was approximately 1.7-fold higher than that in P388 cells (Fig. 2A) and glucose consumption in the IDA-resistant cell line increased about 1.9-fold relatively to the parent cell line (Fig. 2B).

Effect of 2-DG and Tm on cell proliferation. We next compared the cytotoxicity of the glycolysis inhibitor 2-DG on both cell lines. The $\mathrm{IC}_{50}$ of 2-DG on P388 was higher than the tested concentrations ( $>500 \mu \mathrm{M})$, while on P388/IDA cells was 392.6 $\pm 41.1 \mu \mathrm{M}$ (Fig. 3A). 2-DG both suppressed glycolysis and induced ER stress. To examine the impact of ER stress on the cells, the effect of Tm was assessed. The $\mathrm{IC}_{50}$ of Tm on P388 and P388/IDA cells was 9.2 \pm 1.6 and $16.8 \pm 2.4 \mathrm{ng} / \mathrm{ml}(\mathrm{P}<0.05)$, respectively (Fig. 3B). The effect of Tm on P388 cells was about 1.8-fold higher than that in P388/IDA cells.

2-DG enhanced the cytotoxic effect of IDA on P388/IDA cells. Simultaneous treatment of P388/IDA cells with $250 \mu \mathrm{M} 2-\mathrm{DG}$ and $0.5 \mu \mathrm{M}$ IDA enhanced cell death in comparison with each agent alone (Fig. 4A). The expression of cleaved caspase-3 and cleaved PARP was also increased in co-treated cells (Fig. 4B).

\section{Discussion}

IDA-resistance in acute leukemia poses a critical problem for cancer chemotherapy. One of the reasons for the development of resistance to anthracycline anticancer drugs is the overexpression of $\mathrm{P}$-gp in cancer cells $(4,5)$. We found that $\mathrm{P}$-gp was overexpressed in P388/IDA cells compared to P388 cells (data not shown). However, the highly lipophilic drug IDA is less affected by $\mathrm{P}$-gp expression than other anthracyclines $(7,8)$. Therefore, targeting P-gp to overcome IDA-resistance in leukemia is not an efficient strategy. Metabolic alteration, such as through activation of the glycolysis pathway, in drug-resistant cancers has been the focus of potential cancer therapies (19). While it is known that the expression of genes related to glucose metabolism are upregulated in drug-resistant cancer cells compared to parent cells $(19,20)$, the effect of metabolic alteration on drug resistance is poorly understood. In this paper, IDA-resistant P388 leukemia cells increased their dependence on glycolysis for ATP production, as demonstrated by the increased lactic acid production and glucose consumption. Therefore, we expected the glycolysis inhibitor 2-DG to positively affect IDA-resistant P388 cells. It has been reported that 2-DG exerts synergistic cytotoxic effects with other reagents (21). In P388/IDA cells, the combination of IDA $(0.1 \mu \mathrm{M})$ with 2 -DG $(100 \mu \mathrm{M})$, which are concentrations causing low levels of toxicity, could not enhance cell death (data not shown). The cytotoxicity of this combination is thought to be an additive effect, not a synergistic effect.

Additionally, cytotoxicity of $500 \mu \mathrm{M} 2-\mathrm{DG}$ on P388 cells was low, but 2-DG elicited cytotoxic effects at lower concentrations on P388/IDA cells. On the other hand, the ER stress inducer Tm induced higher cytotoxicity in P388 cells than in
P388/IDA cells. These results suggest that P388/IDA cells are resistant to ER stress, and the dominant effect of 2-DG on P388/IDA cells is exerted through glycolysis inhibition. Therefore, targeting the glycolytic pathway on IDA-resistant leukemia cells could be a useful strategy to overcome drug-resistance. However, clinical studies on the application 2-DG in cancers have reported that 2-DG administration induces several side effects, such as fatigue and dizziness, among others $(22,23)$. Additionally, the effective concentration of 2-DG on cancer cells has been found to vary across different cancer cells $(11,19,24,25)$. The underlying mechanism of such differences is poorly understood and the administration of high concentrations of 2-DG increases the risk of side effects. To establish an effective cancer therapy strategy using glycolysis inhibitors, further studies focusing on reliable markers of effective cytotoxicity induced by glycolysis inhibition are necessary.

In conclusion, this study demonstrated that the glycolysis inhibitor 2-DG enhances IDA-induced cell death in P388/IDA leukemia cells, in which glycolytic metabolism is increased. A therapeutic combination of IDA and 2-DG might be a potential strategy against IDA-resistant leukemia.

\section{Acknowledgements}

Not applicable.

\section{Funding}

No funding was received.

\section{Availability of data and materials}

The datasets used and/or analyzed in the present study are available from the corresponding author on reasonable request.

\section{Authors' contributions}

TM conceived and designed the experiments. TM, YK and EH performed the experiments and analyzed the data. TM and YS wrote the manuscript. YS interpreted the data. All authors read and approved the final manuscript.

\section{Ethics approval and consent to participate}

Not applicable.

\section{Patient consent for publication}

Not applicable.

\section{Competing interests}

The authors declare that they have no competing interests.

\section{References}

1. Gewirtz DA: A critical evaluation of the mechanisms of action proposed for the antitumor effects of the anthracycline antibiotics adriamycin and daunorubicin. Biochem Pharmacol 57: 727-741, 1999. 
2. Minotti G, Menna P, Salvatorelli E, Cairo G and Gianni L: Anthracyclines: Molecular advances and pharmacologic developments in antitumor activity and cardiotoxicity. Pharmacol Rev 56: 185-229, 2004

3. McGowan JV, Chung R, Maulik A, Piotrowska I, Walker JM and Yellon DM: Anthracycline chemotherapy and cardiotoxicity. Cardiovasc Drugs Ther 31: 63-75, 2017.

4. Nooter K, Sonneveld P, Oostrum R, Herweijer H, Hagenbeek T and Valerio D: Overexpression of the mdrl gene in blast cells from patients with acute myelocytic leukemia is associated with decreased anthracycline accumulation that can be restored by cyclosporin-A. Int J Cancer 45: 263-268, 1990.

5. Berman E and McBride M: Comparative cellular pharmacology of daunorubicin and idarubicin in human multidrug-resistant leukemia cells. Blood 79: 3267-3273, 1992.

6. Zhou XW, Xia YZ, Zhang YL, Luo JG, Han C, Zhang H, Zhang C, Yang L and Kong LY: Tomentodione M sensitizes multidrug resistant cancer cells by decreasing P-glycoprotein via inhibition of p38 MAPK signaling. Oncotarget 8: 101965-101983, 2017.

7. Mankhetkorn S, Dubru F, Hesschenbrouck J, Fiallo M and Garnier-Suillerot A: Relation among the resistance factor kinetics of uptake, and kinetics of the P-glycoprotein-mediated efflux of doxorubicin, daunorubicin, 8-(S)-fluoroidarubicin, and idarubicin in multidrug-resistant K562 cells. Mol Pharmacol 49: 532-539, 1996

8. Roovers DJ, van Vliet M, Bloem AC and Lokhorst HM Idarubicin overcomes P-glycoprotein-related multidrug resistance: Comparison with doxorubicin and daunorubicin in human multiple myeloma cell lines. Leuk Res 23: 539-548, 1999

9. Vander Heiden MG, Cantley LC and Thompson CB: Understanding the Warburg effect: The metabolic requirements of cell proliferation. Science 324: 1029-1033, 2009.

10. Orang AV, Petersen J, McKinnon RA and Michael MZ: Micromanaging aerobic respiration and glycolysis in cancer cells. Mol Metab 23: 98-126, 2019.

11. Gu L, Yi Z, Zhang Y, Ma Z, Zhu Y and Gao J: Low dose of 2-deoxy-D-glucose kills acute lymphoblastic leukemia cells and reverses glucocorticoid resistance via $\mathrm{N}$-linked glycosylation inhibition under normoxia. Oncotarget 8: 30978-30991, 2017.

12. Pelicano H, Martin DS, Xu RH and Huang P: Glycolysis inhibition for anticancer treatment. Oncogene 25: 4633-4646, 2006.

13. Garg AD, Maes $\mathrm{H}$, van Vliet AR and Agostinis P: Targeting the hallmarks of cancer with therapy-induced endoplasmic reticulum (ER) stress. Mol Cell Oncol 2: e975089, 2014.

14. DeSalvo J, Kuznetsov JN, Du J, Leclerc GM, Leclerc GJ, Lampidis TJ and Barredo JC: Inhibition of Akt potentiates 2-DG-induced apoptosis via downregulation of UPR in acute lymphoblastic leukemia. Mol Cancer Res 10: 969-978, 2012.
15. Badiner GJ, Moy BC, Smith KS, Tarpley WG, Groppi VE and Bhuyan BK: P388 leukaemia cells resistant to the anthracycline menogaril lack multidrug resistant phenotype. Br J Cancer 62: 378-384, 1990

16. Kiue A, Sano T, Naito A, Inada H, Suzuki K, Okumura M, Kikuchi J, Sato S, Takano H, Kohno K, et al: Reversal by two dihydropyridine compounds of resistance to multiple anticancer agents in mouse P388 leukemia in vivo and in vitro. Jpn J Cancer Res 81: 1057-1064, 1990.

17. Aboudkhil S, Henry L, Zaid A and Bureau JP: Effect of testosterone on growth of P388 leukemia cell line in vivo and in vitro. Distribution of peripheral blood T lymphocytes and cell cycle progression. Neoplasma 52: 260-266, 2005.

18. Matsuo T and Sadzuka Y: Extracellular acidification by lactic acid suppresses glucose deprivation-induced cell death and autophagy in B16 melanoma cells. Biochem Biophys Res Commun 496: 1357-1361, 2018.

19. Song K, Li M, Xu X, Xuan LI, Huang G and Liu Q: Resistance to chemotherapy is associated with altered glucose metabolism in acute myeloid leukemia. Oncol Lett 12: 334-342, 2016.

20. Staubert C, Bhuiyan H, Lindahl A, Broom OJ, Zhu Y, Islam S, Linnarsson S, Lehtiö J and Nordström A: Rewired metabolism in drug-resistant leukemia cells: A metabolic switch hallmarked by reduced dependence on exogenous glutamine. J Biol Chem 290 8348-8359, 2015

21. Miwa H, Shikami M, Goto M, Mizuno S, Takahashi M, Tsunekawa-Imai N, Ishikawa T, Mizutani M,Horio T, Gotou M, et al: Leukemia cells demonstrate a different metabolic perturbation provoked by 2-deoxyglucose. Oncol Rep 29: 2053-2057, 2013.

22. Raez LE, Papadopoulos K, Ricart AD, Chiorean EG, Dipaola RS, Stein MN, Rocha Lima CM, Schlesselman JJ, Tolba K, Langmuir VK, et al: A phase I dose-escalation trial of 2-deoxy-D-glucose alone or combined with docetaxel in patients with advanced solid tumors. Cancer Chemother Pharmacol 71 : 523-530, 2013.

23. Stein M, Lin H, Jeyamohan C, Dvorzhinski D, Gounder M, Bray K, Eddy S, Goodin S, White E and Dipaola RS: Targeting tumor metabolism with 2-deoxyglucose in patients with castrate-resistant prostate cancer and advanced malignancies. Prostate 70: 1388-1394, 2010

24. Maximchik P, Abdrakhmanov A, Inozemtseva E, Tyurin-Kuzmin PA, Zhivotovsky B and Gogvadze V: 2-Deoxy-D-glucose has distinct and cell line-specific effects on the survival of different cancer cells upon antitumor drug treatment. FEBS J 285: 4590-4601, 2018.

25. Reyes R, Wani NA, Ghoshal K, Jacob ST and Motiwala T: Sorafenib and 2-deoxyglucose synergistically inhibit proliferation of both sorafenib-sensitive and -resistant HCC cells by inhibiting ATP production. Gene Expr 17: 129-140, 2017. 